\title{
Patient voice in Core Outcome Sets: Are we hearing but not listening?
}

\author{
Sanne Gordijn ${ }^{1}$ and Wessel Ganzevoort ${ }^{2}$ \\ ${ }^{1} \mathrm{UMCG}$ \\ ${ }^{2}$ Amsterdam UMC - Locatie AMC
}

May 21, 2021

BJOG commentary for BJOG-21-0466 A core outcome set for the treatment of pregnant women with pregestational diabetes: an international consensus study

\section{Sanne J Gordijn ${ }^{1}$, Wessel Ganzevoort ${ }^{2}$}

${ }^{1}$ Obstetrics and Gynecology, University Medical Center Groningen, University of Groningen, Groningen, The Netherlands

${ }^{2}$ Obstetrics and Gynecology, Amsterdam University Medical Centers, Amsterdam, University of Amsterdam, The Netherlands

The authors report no conflict of interest.

Title: Patient voice in Core Outcome Sets: Are we hearing but not listening?

The article by Kgosidialwa O. et al. is a good example of the process of uniformization of research ${ }^{1}$. In the absence of unambiguous empirical evidence, a (modified) Delphi consensus procedure is the method of choice to develop agreement on definitions, core outcome sets, and other sets of relevant items. The Delphi procedure taps into the contemporary knowledge and values of individual participants, including lay experts and patient representatives ${ }^{2}$. Patient involvement is advocated because they are the primary stakeholders and best informed about what outcomes are most important to them. An example: the perception of the importance of breast feeding as an outcome of obstetric intervention studies may differ between patients and health care providers.

The COMET initiative provides tools for COS development, and initiated a working group for patient involvement ('People and Patient Participation, Involvement and Engagement (PoPPIE)'). A full understanding of the concept of the COS itself for all stakeholders and particularly for patients is of paramount importance and promoted by a video explaining the COS concept for patients and other stakeholders ${ }^{3}$.

It is important to actively counteract the effects of biases such as heterogeneity in knowledge and numbers among stakeholder groups and carefully balance participants between stakeholder groups in order to allow for minority opinions and prevent attrition bias (thereby overestimating agreement).

In our own experience in COS development procedures, several issues complicate patient involvement. This is seen in many studies, including the one by Kgosidialwa O. et al. ${ }^{1}$ :

The patient group is typically smaller than the group of professional experts. In the current study, 46/205 (22\%) participants were patients in the first round and $2 / 26(8 \%)$ of the participants were in the consensus meeting. Also, no stratified responses were analysed (which would also pose challenges for semi anonymity). Thus, the patient voice may not be sufficiently heard among other stakeholders. We wonder: should the 
patient have a stronger voice than the other stakeholders? The COMET methodology does not give guidance on prioritized inclusion of outcomes that have a very high percentage agreement (for example $>90 \%$ ) in specific predefined stakeholder groups (like patients). To illustrate the point: of 21 outcomes scoring $>90 \%$ among patients in the first round only 5 were included in the COS and of 20 outcomes scoring $>90 \%$ by the professionals 16 were included in the COS: patient preferences are numerically undervalued.

In a consensus meeting, individuals from professional stakeholder groups are all highly educated and experienced caregivers or researchers. Patient participants, often recruited from patient organisations, also have a selection towards higher education, but their experience of the condition is usually personal. Given the obvious difference in medical expertise means patients will be influenced by the professional stakeholders. In the example above, 14 of 21 outcomes that scored $>90 \%$ in the patient group were excluded during the consensus meeting.

Consensus procedures clearly have the potential to bring research forward in many areas. This has increased uniformity and fostered collaboration. We applaud that it has become self-evident that involvement of the primary stakeholders is key. However, in our view this needs to be more than a pretence: it is time to evaluate if the mechanics of these procedures adequately allow the perspective of what patients find valuable to be incorporated.

1. Kgosidialwa O, Bogdanet D, Egan AM, et al. A core outcome set for the treatment of pregnant women with pregestational diabetes: an international consensus study. BJOG: An International Journal of Obstetrics $\mathcal{E}$ Gynaecology. 2021.

2. Williamson PR, Altman DG, Bagley H, et al. The COMET Handbook: version 1.0. Trials. 2017;18(Suppl 3):280.

3. COMET. The COMET Initiative's Guide to Selecting Outcomes in Clinical Trials. https://youtu.be/Hpmtk4v5xpA. Published 2018. Accessed. 